\title{
Acute Lymphoblastic Leukemia in Children
}

Treatment Planning via Minimal Residual Disease Assessment

Claus R. Bartram, André Schrauder, Rolf Köhler, Martin Schrappe

\section{SUMMARY}

Background: Acute lymphoblastic leukemia (ALL) is the most common type of cancer in children and adolescents, accounting for $30 \%$ of all cases of malignancy in this age group. The cure rate of ALL is now above $80 \%$. The clinical and biological characteristics of ALL that have been studied to date are of limited use in predicting the individual response. Newly developed methods for the assessment of minimal residual disease (MRD) are more helpful in this regard.

Methods: Review of pertinent literature retrieved by a selective search in Medline.

Results: MRD assessment has gradually been incorporated into ALL treatment planning over the past two decades. In the largest study to date of the use of MRD for this purpose, which included 3648 children with ALL, the MRD status on days 33 and 78 after the start of treatment was found to be the most important prognostic factor. The study group included 3184 patients with B-precursor ALL (leukemia consisting of immature B-lymphocytes), of whom a large subgroup (standard risk profile, 42\%) had a seven-year event-free survival rate (7Y-EFS) of $91.1 \%$; for the $6 \%$ of B-ALL patients with a high-risk profile, the cumulative rate of recurrence was $38.5 \%$.The remaining 464 patients had T-ALL (leukemia consisting of T-lymphocytes). The leukemia cells were eliminated more slowly overall in these patients than in those with B-ALL. Nonetheless, the T-ALL patients with a standard risk profile ( $16 \%$ of all T-ALL patients) had an excellent 7 Y-EFS rate $(91.1 \%)$, while the high-risk group $(21 \%$ of all T-ALL patients) had an MRD recurrence rate of $37.7 \%$. These findings are representative of current data from around the world on children and adults with ALL.

Conclusion: MRD analysis enables more accurate prediction of ALL patients' response to treatment. Risk-group stratification by MRD assessment has already brought about considerable improvement in individualized treatment planning.

\section{- Cite this as:}

Bartram CR, Schrauder A, Köhler R, Schrappe M: Acute lymphoblastic leukemia in children - treatment planning via minimal residual disease assessment. Dtsch Arztebl Int 2012; 109(40): 652-8.

D0I: 10.3238/arztebl.2012.0652

\begin{abstract}
1 cute lymphoblastic leukemia (ALL) is the $\Delta$ commonest malignancy in children (aged 1-18 years), accounting for almost $30 \%$ of all cancers in this age group. In Germany, every year four in every 100000 children aged up to 15 develop ALL (Box). Almost all patients in Germany are treated according to the valid treatment algorithms developed by the two collaborative study groups ALL-BFM (Berlin-Frankfurt-Münster) and COALL (cooperative study group for childhood acute lymphoblastic leukemia), which are subdivided into three treatment phases (induction and consolidation of remission, re-intensification, maintenance treatment) and cover two years. Notable improvements in the treatment of childhood ALL have been made in the past four decades (1-6); compared with the late $1960 \mathrm{~s}$, when $30 \%$ of cases were cured, nowadays some $80 \%$ of patients remain in their first remission even after 10 years. The remaining $20 \%$ of children have recurrences, and the cure rate falls to 25-40\%. Conversely, it is also subject to discussion that a proportion of the cured patients are currently overtreated and would benefit from a reduction in treatment for the sake of lower toxicity and fewer long-term sequelae. In addition to determining patient-specific risk factors (such as sex, age) and ALL-specific risk factors (such as leukocyte count, immunophenotype, chromosomal aberrations) when making the diagnosis, in vivo confirmation of responsiveness to chemotherapy has been found to be of particular relevance in predicting the likelihood of survival and risk of recurrence (7-9).
\end{abstract}

The standard method to gauge the therapeutic response is cytomorphology by means of microscopy. Its disadvantage is its low sensitivity, which in the bestcase scenario allows detection of one leukemia cell among 100 normal cells. According to this diagnostic criterion, 95-98\% of patients achieve complete remission within four weeks of starting therapy. This means that in substantial proportions, intensive polychemotherapy was administered after the leukemia had become undetectable. Two decades ago, a diagnostic breakthrough was reached when polymerase chain reaction (PCR) was introduced to identify minute amounts of malignant cells (minimal residual disease, MRD). This review article will highlight the great importance of MRD analysis for tailoring individual therapeutic approaches in children with ALL. The authors' own results were supplemented with a 
selective Medline search using the search terms "PCRMRD", "childhood ALL", and with the therapeutic studies BFM (Berlin- Frankfurt-Münster) und AIEOP (Assoziazone Italiana Ematologia Oncologia Pediatrica).

\section{Methods for diagnosing MRD}

Three methods for identifying minute amounts of malignant cells (minimal residual disease, MRD) allow the identification of one leukemia cell among 10000 to 1 million normal cells $\left(10^{-4}\right.$ to $\left.10^{-6}\right)$ and are thus 100-10 000 times more sensitive than conventional cytomorphological techniques. These methods differ with regard to their applicability (frequency of relevant target structures) in terms of their sensitivity and their expense in terms of time and money (10):

- Immunophenotyping using flow cytometry and multiple markers to identify leukemia-specific immunophenotypes,

- PCR analysis of fusion products of leukemiaspecific chromosomal translocations (for example, BCR-ABL recombination in the context of the Philadelphia translocation-t $[9 ; 22])$, and

- PCR analysis of clone-specific chain joining regions of immunoglobulin (Ig) and T-cell receptor (TCR) rearrangements.

In the meantime the third method in this list has become most widespread in Europe in the context of European ALL studies because of its excellent sensitivity. This method is based on the insight that ALL always starts from individual lymphocyte precursors, and all leukemias are therefore characterized by an individual, clone-specific pattern on Ig rearrangements or TCR rearrangements.

The molecular basis is a DNA rearrangement in which different segments of the Ig genes or TCR genes are joined individually (11). In the context of these recombination processes, fewer or more nucleotides from the original DNA sequence are lost at the joining regions, and free nucleotides can be inserted into this region. The result is a unique DNA sequence for a particular lymphocyte, which can be identified on the basis of PCR analysis by using allele-specific DNA probes - a method that the authors and other working groups have been developing since the late 1980s in order to measure MDR in patients with $\operatorname{ALL}(12,13)$.

Since acute lymphoblastic leukemia can display subpopulations of an initial leukemia cell clone (oligoclonality) at the time of diagnosis and may undergo further Ig- and TCR-rearrangements during the disease course, which may then result in the loss of the DNA sequences used as probes, it is recommended for the PCR analysis to use two or more target sequences in order to avoid false-negative results. In the context of the BFM-ALL study, 51 PCR primer combinations are currently used for every patient, so as to be able to identify the IgH-, IgK-, TCR $\beta$-, TCR $\gamma$ - und TCR $\delta$-recombinations that are characteristic of the respective leukemia. These joining regions are subsequently sequenced, and allelespecific oligonucleotide primers are generated on this basis, which are then examined for their sensitivity and

\section{Acute lymphoblastic leukemia (ALL) in children (basic facts)}

- ALL is the most common childhood cancer and affects 600 new patients in Germany every year.

- The clinical presentation is characterized by the consequences of leukemic metaplasia of the bone marrow and possible organ involvement:

- Severe infections (because of absolute granulocytopenia),

- Hemorrhage or tendency to hemorrhage (because of thrombopenia),

- Anemia,

- Dyspnea (mediastinal tumor, especially in T-cell leukemia)

- Pain (especially in the legs, "the child does not want to walk any further"), and

- Abdominal problems: hepatosplenomegaly.

- Treatment is based on polychemotherapy tailored by risk of recurrence; substances administered include corticosteroids, metaphase inhibitors (vinca alkaloids), asparaginase (depletion of asparagine), anthracyclines, alkylating agents, antimetabolites (methotrexate), purine antagonists (mercaptopurin). Skull radiotherapy was the only neurological therapy in the past and has in the meantime been replaced with intrathecal therapy for most patients. Allogenic blood stem cell transplantation is of great importance especially in patients at high risk or after a recurrence.

- ALL is fatal if untreated.

\section{FIGURE 1}

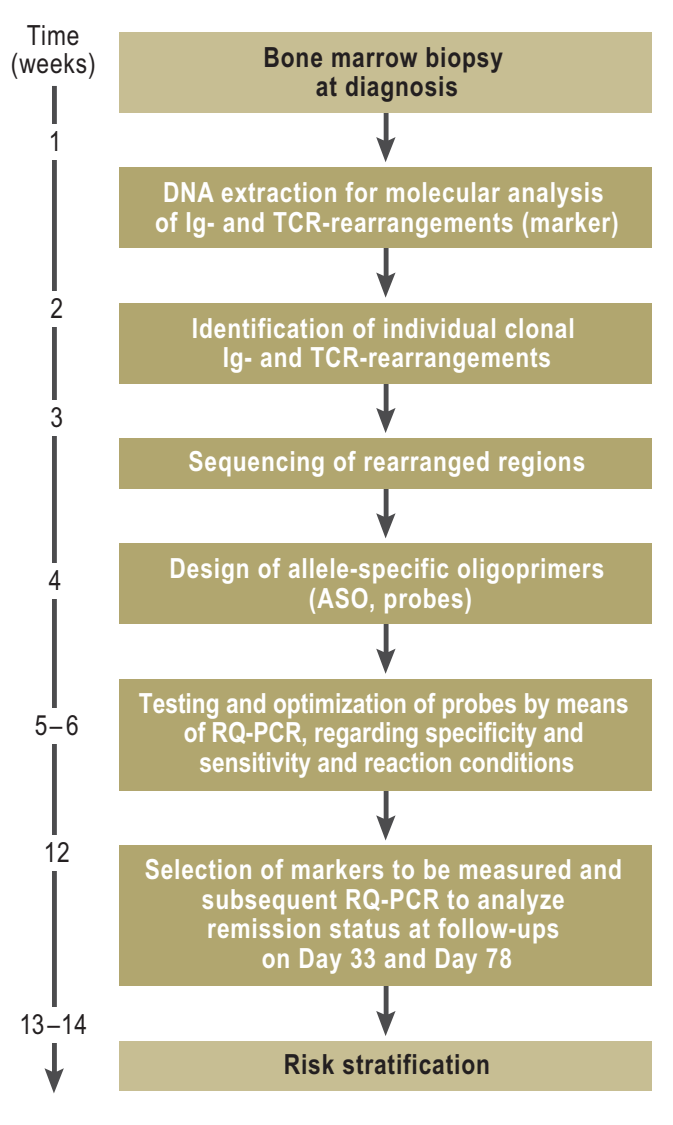

Individual steps of MRD analysis in sequence (in weeks), from diagnosis to stratification into MRDbased risk groups. MRD $=$ minimal residual disease 
RQ-PCR analysis of an ALL patient with an detection were determined in a dilution series of $10^{-1}$ to $10^{-5}$ with one leukemia cell per 100000 normal cells $\left(10^{-5}\right)$. The threshold value describes the start of the exponential phase of PCR. At time points R1 (light brown, Day 33) and R2 (red, Day 78), $10^{-3}$ and $10^{-4}$ residual leukemia cells are found, which corresponds to intermediate risk allele-specific IgH oligomere whose limits of

\section{FIGURE 2}

Intensity

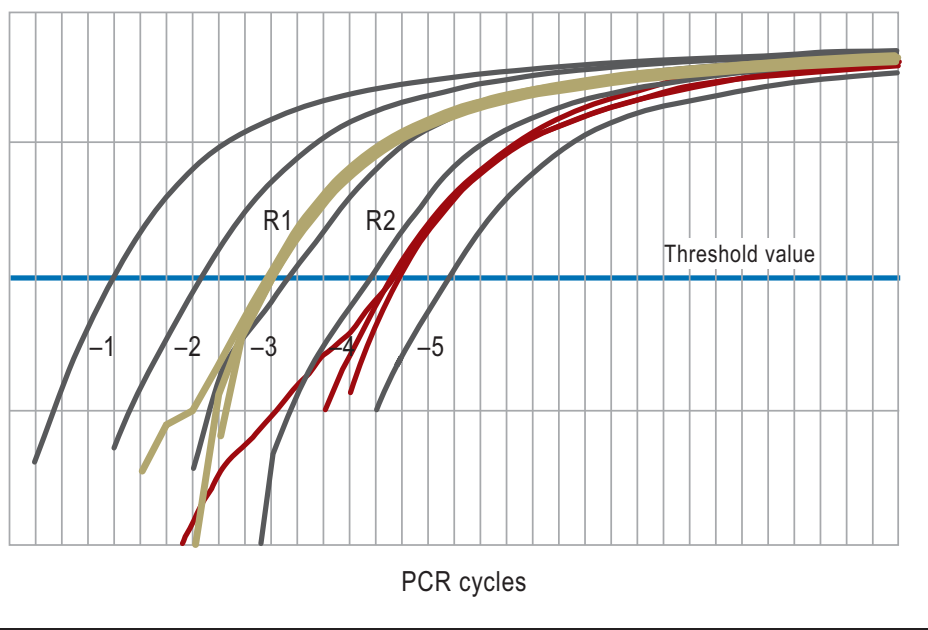

\section{FIGURE 3}

B) cumulative incidences (Cl) of recurrence after MRD-based stratification of 3184 patients with precursor-B-ALL (the results were originally published in Blood/ American Society of Hematology (Conter V, Bartram CR, Valsecchi MG, et al.: Molecular response to treatment redefines all prognostic factors in children and adolescents with B-cell precursor acute lymphoblastic leukemia: results in 3184 patients of the AIEOP-BFM ALL 2000 study. Blood 2010; 22: 3206-14)

$\mathrm{SR}=$ standard risk, $\mathrm{IR}=$ intermediate risk, $\mathrm{HR}=$ high risk

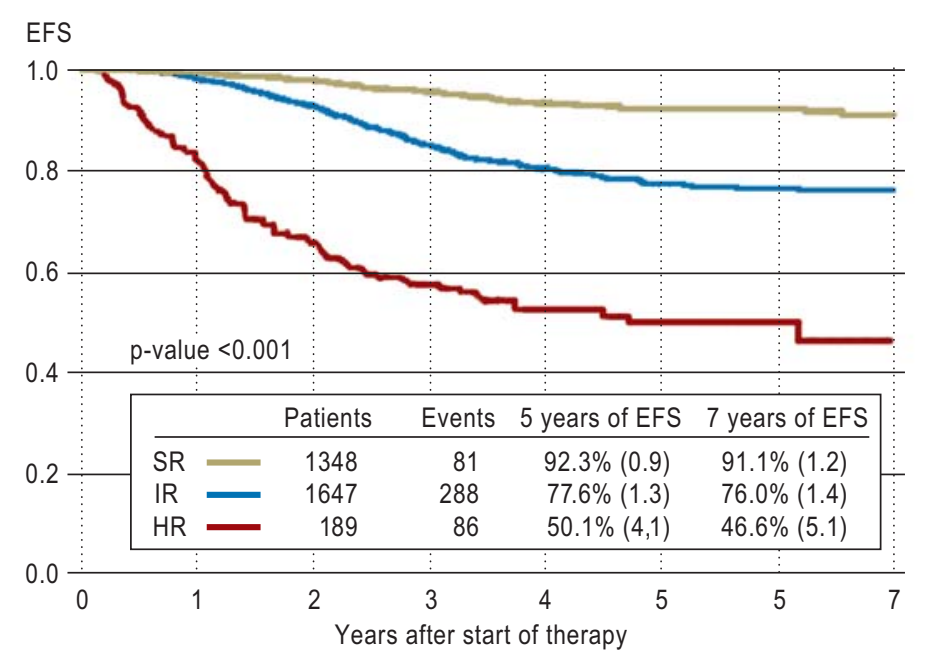

Cumulative incidence
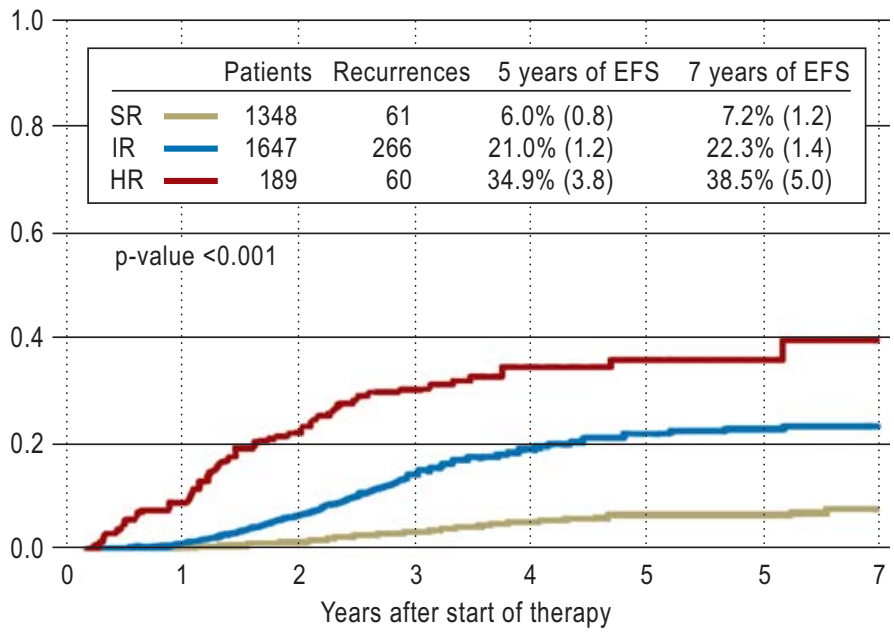
specificity by using real-time quantitative PCR (RQPCR). This is mostly done by using serial dilutions, in which DNA amplificates of the leukemia cells are placed in control DNA of normal cells in dilutions of $10^{-1}$ to $10^{-5}$ and the limits are determined at which leukemia cells can be detected. Finally, RQ-PCR is used to determine the remission status at patients' follow-ups. Figure 1 shows the time sequence from initial diagnosis to MRD-based risk stratification three months after the start of treatment.

\section{The long road towards clinical application}

The application of MRD analysis in the treatment of childhood ALL that is under discussion in this article provides an example of the careful, evidence-based introduction of new methods and marker systems of molecular medicine into clinical routine. The BFM Study Group recognized the clinical potential of this technique early on, which was developed further over the years, and in the early 1990s included a relevant clinical research project in the ALL-BFM 90 study protocol. From the very beginning, the emphasis was put on international agreement in the setting of the International BFM Study Group. Since the kinetics of residual leukemia cells under MRD criteria was not known, and neither was their clinical relevance, researchers agreed after extensive discussions (of the ethical implications in particular) that, after providing adequate information to and obtaining written consent from the participants, bone marrow specimens would be harvested (under general anesthesia) at nine time points during and after the end of the treatment, so as to determine the MRD status and correlate this with the clinical data. The results were evaluated retrospectively and during the project phase made available neither to the treating physicians nor to the patients, in order to prevent decisions that were not based on evidence.

The findings obtained from 240 patients were available in 1998 (14) and were confirmed simultaneously by other groups (15). MRD analysis was found to be superior to all conventional prognostic parameters. Puncture timings on days 33 and 78 after the start of treatment were of particular prognostic relevance; they provided essential indications of a positive clinical course or high risk of recurrence and made it feasible to restrict follow-up studies to these two control points. According to MRD criteria, three risk groups became obvious: patients at very low risk of recurrence of only $2 \%$ ( $43 \%$ of children included in the study), those with a very high risk of recurrence of $75 \%$ ( $15 \%$ of cases), and an intermediate group with a recurrence rate of $23 \%$ ( $43 \%$ of patients). These findings were still valid after 10 years of follow-up (16). Such a clear distinction of the different risk profiles had previously not been available. Also of note: Compared with conventional prognostic criteria, relevant shifts were observed in the individual risk profiles: Patients who had previously been allocated to the standard risk group were at intermediate or high risk of recurrence when MRD criteria were used, and vice versa.

\section{TABLE}

\section{Definition of risk groups}

\begin{tabular}{|c|c|c|c|c|}
\hline \multirow{3}{*}{$\begin{array}{l}\text { Risk- } \\
\text { group } \\
\text { SR }\end{array}$} & \multicolumn{4}{|c|}{ Definition of risk groups } \\
\hline & \multicolumn{2}{|l|}{ ALL-BFM 95} & \multicolumn{2}{|c|}{ AIEOP-BFM ALL 2000} \\
\hline & $\begin{array}{l}\text { Leukocytes } \\
<20000 / \mu \mathrm{L} \text {, and } \\
\text { age } 1-5 \text { years }\end{array}$ & \multirow{2}{*}{$\begin{array}{l}\text { PRED-GR*1, } \\
\text { and } \\
\text { CR Day } 33^{* 2} \\
\text { and } \\
\text { no detection of } \\
t(4 ; 11) \\
\text { or } t(9 ; 22)\end{array}$} & $\begin{array}{l}\text { MRD negative } \\
\text { Days } 33 \text { and } \\
78^{* 3}\end{array}$ & \multirow{2}{*}{$\begin{array}{l}\text { PRED-GR* } \\
\text { and } \\
\text { CR Day } 33^{* 2} \\
\text { and } \\
\text { no detection o } \\
\mathrm{t}(4 ; 11) \\
\text { or } \mathrm{t}(9 ; 22)\end{array}$} \\
\hline MR & $\begin{array}{l}\text { Leukocytes } \\
\geq 20000 / \mu \mathrm{L}, \text { or } \\
\text { age } \geq 6 \text { years }\end{array}$ & & $\begin{array}{l}\text { MRD positive, } \\
\text { on Day } 78 \text { but } \\
<10-3\end{array}$ & \\
\hline$H R$ & \multicolumn{2}{|c|}{$\begin{array}{l}\text { PRED-PR }{ }^{* 1} \text {, or } \\
\text { no CR on Day } 33 \text {, or } \\
\text { translocation } t(4 ; 11) \text { or } t(9 ; 22)\end{array}$} & \multicolumn{2}{|c|}{$\begin{array}{l}\text { MRD on Day } 78 \geq 10^{-3} \text {, or one of } \\
\text { the HR criteria from } \\
\text { ALL-BFM } 95 \text { Study }\end{array}$} \\
\hline
\end{tabular}

${ }^{* 1}$ Prednisone response: If the cytological assessment of the proportion of lymphoblasts in the blood specimen after the first week of therapy (only prednisone $60 \mathrm{mg} / \mathrm{m}^{2} / \mathrm{d}$ and one dose of intrathecal methotrexate) shows a number of below 1000 lymphoblasts/ $\mathrm{LL}$, the term used is that of Prednisone Good Response (PRED-GR); for 1000 lymphoblasts or more, the term is Prednisone Poor Response (PRED-PR)

${ }^{*} \mathrm{CR}$ : complete remission; after 32 days of induction therapy, bone marrow biopsy is planned to determine cytological remission (M1 marrow, less than $5 \%$ of lymphoblasts); the specimen for the first MRD assessment is harvested at the same time.

${ }^{* 3}$ No detection of MRD with a sensitivity of at least two molecular targets with at least $1 \times 10^{-4}$.

$\mathrm{SR}=$ standard risk, $\mathrm{IR}$ = intermediate risk, $\mathrm{HR}=$ high risk; $\mathrm{MRD}=$ minimal residual disease

\section{FIGURE 4}

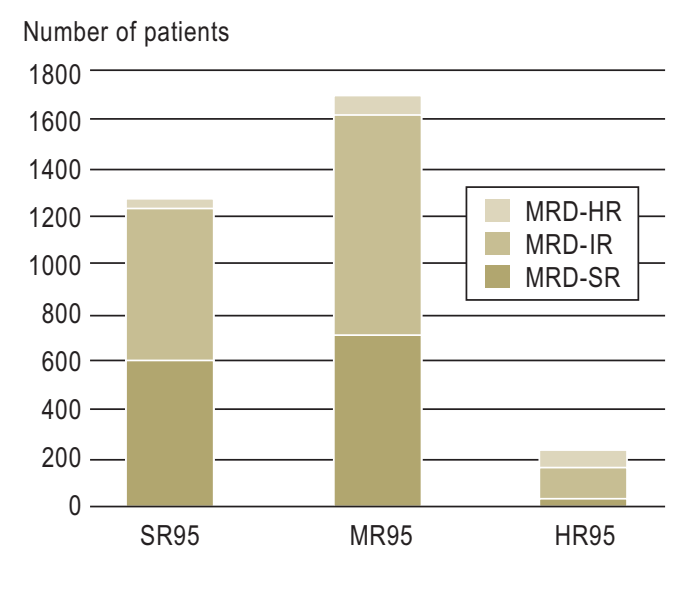

\section{From pilot project to general MRD-based therapeutic stratification}

On the basis of these findings, a prospective therapeutic study of the Associazione Italiana di Ematologia Oncologia Pediatrica and the BFM-ALL Group (AIEOPBFM ALL 2000) was set up that introduced for the first time the MRD-based risk stratification with only two remission samples, and which included some 4700 patients between 2000 and 2006. From the start, the internationally accepted standardization of this analytic technique was driven forward in this context $(10,17$, 18), which was quality assured by means of regular round-robin tests. The evaluation of this globally largest study of its kind proved the logistical feasibility
Redistribution of all patients with precursor-B-ALL in the MRD-based study AIEOP-BFMALL 2000, after applying the "classic" criteria-for example in the ALLBFM-95 Study (standard risk: SR 95; intermediate risk: MR 95; high risk: HR 95 [see Table). 


\section{FIGURE 5}

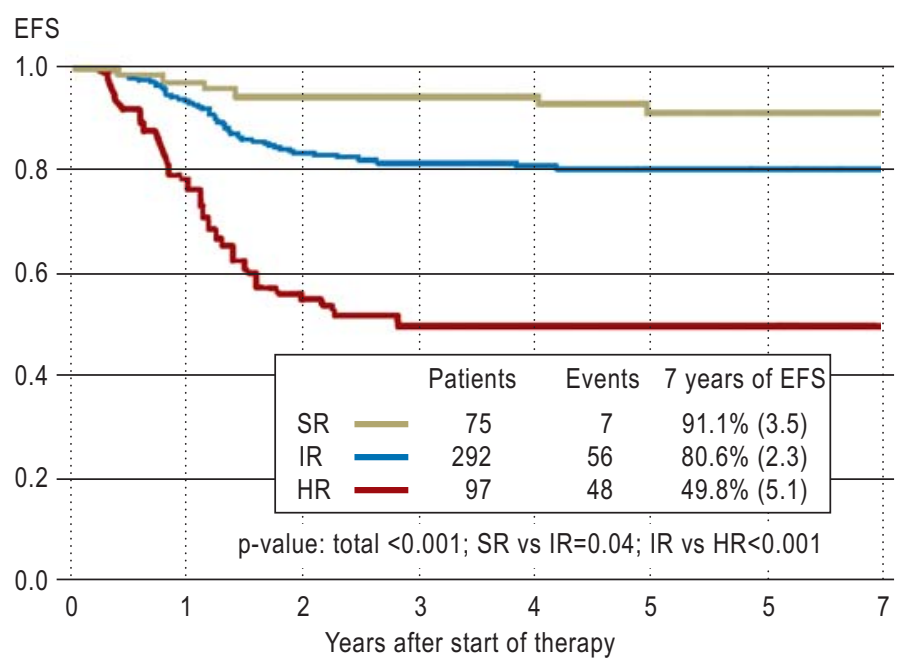

Cumulative incidence

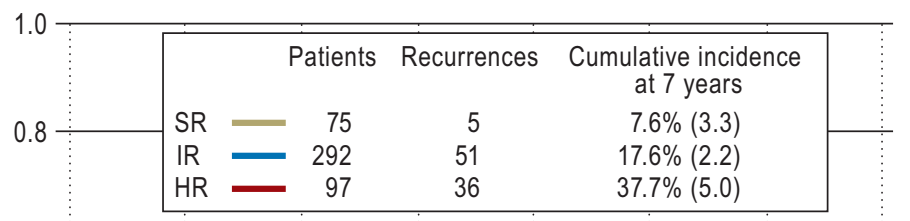

$0.6 \quad$ p-value: total $<0.001 ;$ SR vs IR=0.02; IR vs $H R<0.001$

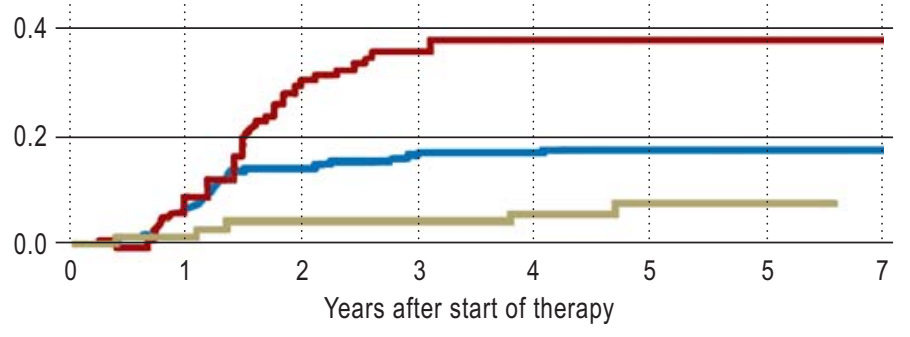

A) Event-free survival (EFS) and

B) cumulative incidence in recurrences after MRD-stratification of 464 patients with T-ALL (the results were originally published in Blood/American Society of Hematology [Schrappe M, Valsecchi MG, Bartram CR, et al.: Late MRD response determines relapse risk overall and in subsets of childhood T-cell ALL: results of the AIEOP-BFM-ALL 2000 study. Blood 2011; 118: 2077-84]).

$\mathrm{SR}=$ standard risk, $\mathrm{IR}=$ intermediate risk, $\mathrm{HR}=$ high risk

of this complex analytic technique in an international multicenter therapeutic study (16). MRD stratification using two or more sensitive markers that enabled detection of a leukemia cell among at least 10000 normal cells $\left(\leq 10^{-4}\right)$ was successful in $80 \%$ of patients. At least one sensitive marker was identified in $94 \%$ of B-cell leukemias and $82 \%$ of patients with T-ALL. Patients without an adequate MRD constellation were stratified using conventional parameters. Check points were Days 33 and 78 after the treatment was started; the MRD findings were available from the laboratory 7 to 14 days later, so that stratification into the three risk groups was complete 13 to 14 weeks after starting treatment.
Patients were stratified into the three MRD-based risk groups (SR, IR, HR — standard risk, intermediate risk, high risk) according to the following criteria:

- Patients whose results were MRD-negative at both control points (Day 33 and Day 78) for two independent MRD markers with a sensitivity of $\leq 10^{-4}$ were classified as patients at standard risk (MRD-SR).

- Patients at intermediate risk (MRD-IR) had positive results on one or both control points, with the second MRD assessment value at $<10^{-3}$; Figure 2 shows an example of this.

- Patients with an MRD measurement of $\geq 10^{-3}$ on Day 78 were classified as at high risk (MRD-HR).

In total, 3184 patients with precursor-B-ALL were stratified by using MRD (19). Of these, 42\% (1348) were MRD-SR patients with 7 years' event-free survival (EFS, standard error) of $91.1 \%(1.2 \%), 52 \%$ (1647) were MRD-IR patients with an EFS of $76 \%$ $(1.4 \%)$, and $6 \%(189)$ were MRD-HR patients with an EFS of $46.6 \%(5.1 \%) \quad(p<0.001)$. The cumulative incidence rates of recurrences were similarly diverse (Figure 3).

Direct comparison with the conventional criteria (Table) shows the advantages of MRD-based risk stratification, such as was applied in the ALL-BFM-95 Study (20) (age, leukocyte count, cytogenetics, response to prednisone) (Figure 4). If these patients had been stratified according to conventional criteria, half of the patients at standard risk (SR 95) would have responded with a delay (medium and dark sections of the SR 95 bar) and thus been at higher risk of recurrence, whereas conversely, patients who had been stratified as at high risk according to conventional criteria (HR 95) responded better to treatment than had been assumed (dark and light sections on the HR 95 bar) and had a better prognosis. All three "classic" risk groups include patients at a particularly high risk of recurrence (MRD$\mathrm{HR}$ ). It is obvious that assessing MRD can describe the risk stratification in a more differentiated and precise way by using patient-specific and leukemia-specific risk factors. However, the clinical benefits will fully materialize only when more effective therapeutic elements are used for patients who are characterized by a slower or poor molecular therapeutic response, which are yet to be developed. Another aspect to this is the fact that the expectation that the excellent therapeutic result in the group at standard risk, which is characterized by a rapid response to treatment, can be realized by less aggressive treatment later on, has so far not been confirmed by preliminary results (M Schrappe, personal communication). In the AIEOP-BFM ALL 2000 Study, 464 T-ALL patients were stratified according to the MRD criteria explained above (21): $16 \%$ (75) of the patients were classified as MRD-SR, with a 7 -year EFS of $91.1 \%$; 63\% (292) of patients were classified as MRD-IR, with a 7-year EFS of $80.6 \%$; and $21 \%$ (97) of patients were classified as MRD-HR, with a 7-year EFS of $49.8 \%(\mathrm{p}<0.001)$ (Figure 5). 
It was found that both forms of leukemia had an equally good result (EFS) in the MRD-SR group, independently of the immunophenotype (B-cell or T-cell leukemia). This observation also applied to the other two risk groups. However, it should be noted that the MRD-SR group included a lower proportion of patients with T-ALL than precursor-B-ALL patients, and the distribution in the MRD-HR group is exactly the opposite. In patients with T-ALL the elimination of leukemia cells is therefore on average noticeably delayed compared with precursor-B-ALL. If patients with T-ALL present as MRD negative on Day 33, however, the recurrence rate of $8 \%$ makes for an excellent prognosis (Figure 5) (21). Furthermore, 32\% of all T-ALL patients in the MRD-IR group who were MRD negative on Day 78 also had an excellent result; the seeming conclusion is that MRD positivity on Day 33 is of clinically lesser importance for T-ALL than for precursorB-ALL. A large number of residual leukemia cells $\left(\geq 10^{-3}\right)$ on Day 78 is the most important predictor for recurrence in T-ALL patients.

\section{Conclusions and outlook}

The AIEOP-BFM ALL 2000 Study has set international standards for risk stratification in children with ALL. The MRD-based prognostic estimate has proved superior to all other parameters. This assessment has been confirmed in numerous studies, independently of which method was used. This holds for children $(22,23)$ as well as for adults $(24,25)$ which is notable since the biological basis of the pathogenesis of leukemia-including its molecular genetic pathogenesis - is very different in both age groups. However, it underlines the importance - independently of other factors - of this new therapeutic option.

MRD-supported therapy also has an important role for other hematopoietic neoplasias-for example, in chronic lymphocytic leukemia (26) and acute myeloid leukemia (27). Chronic myeloid leukemia provides a particularly instructive example. After the specific cytogenetic aberration, the Philadelphia chromosome, had been discovered in 1960 and the molecular genetic equivalent, BCR-ABL-recombination, had been figured out in the 1980s, this molecular target structure has been used to make the diagnosis and to guide MRD-based treatment. Furthermore, innovative therapeutic approaches that targeted this particular molecular defect in leukemia cells were developed in the shape of the tyrokinase inhibitors, and the treatment of CLL has been notably improved (28). MRD-status in the blood is measured for solid tumors too. But in this setting, the analytic approach is used for a different purpose: It is used to detect tiny amounts of disseminated tumor cells with the potential for metastasis (29).

MRD analysis in children with ALL was included in the diagnostic repertoire of the BFM-ALL Study that is covered by German statutory health insurers; previously this analytic technique was funded for 15 years by project grants from the German Cancer Aid (Deutsche Krebshilfe). Although it is hardly likely that significant improvement rates can be achieved for the large group of patients with an MRD-SR profile, whose cure rates are higher than $90 \%$, the recurrence rate in the MRD-HR group remains unacceptably high — even though this group comprises only $6 \%$ of patients. The intermediate risk group is also in need of improved therapeutic options. Studies are needed to ascertain whether innovative therapeutic approaches that target the molecular defects in leukemia cells ("targeted therapy") yield similar successes as have been reported for tyrokinase inhibitors in BCR-ABL-positive ALL. It should be borne in mind, however, that risk stratification of patients according to MRD criteria is inherently possible only several months after starting therapy. Whether current research projects of the genome-wide sequence analysis of children with ALL will identify new molecular parameters that would enable improved risk stratification at the time of diagnosis, remains to be seen. Any markers would have to be investigated for their validity in large prospective studies with adequate follow-up times. In the medium term, MRD-based risk stratification of patients will therefore remain the gold standard for all patients with ALL.

\section{Acknowledgement}

The initial development of the method for PCR-based Ig- and TCR-analysis was funded by the DFG, German Research Foundation (Bartram). Profound thanks are due to the German Cancer Aid for long years of comprehensive funding (Bartram) in introducing the MRD analysis. Furthermore, the German Cancer Aid has comprehensively funded the respective ALL-BFM Studies (Schrappe). The following scientists were crucially involved in the development and evaluation of the MRD diagnostic technique in Bartram's working group (until 1995 division of molecular biology, department of pediatrics II, University Medical Center Ulm, subsequently institute of human genetics, University of Heidelberg): T E Hansen-Hagge, S Yokota, T Seriu, M Nakao, and T Flohr. We thank our patients and their families for their participation in the studies and all the doctors, nurses, and medical technicians who were involved. We also thank the study commission and members of our MRD laboratories for conceptual help and for conducting the study. Special thanks go to our international partners of more than two decades; names we wish to mention are $J$ $\mathrm{J}$ M van Dongen (Netherlands), A Biondi and G Masera (Italy), R Panzer-Grümayer and $\mathrm{H}$ Gadner (Austria). We thank the initiator of the ALL-BFM studies, $\mathrm{H}$ Riehm, for his conceptual far-sightedness and emphatic support in introducing MRD analysis as a clinical diagnostic technique.

\section{KEY MESSAGES}

- Procedures to detect minute amounts of malignant cells (minimal residual disease, MRD) enables the identification of one leukemia cell among up to 1 million normal cells and is therefore several orders of magnitude more sensitive than cytomorphology.

- In the context of the BFM Study protocols, MRD diagnosis on Day 33 and Day 78 after the start of treatment is key to treating ALL.

- This parameter was found to be the most important prognostic indicator in children with ALL in the globally largest clinical study using MRD-based risk stratification, AIEOP-BFM ALL 2000.

- In patients with precursor-B-ALL, a large group of patients with a very good chance of cure of more than $90 \%$ can be identified, whereas one-third of patients develop recurrences in the smaller high-risk group

- The MRD-based stratification into three risk groups has been found to be the most important factor to guide therapy in T-ALL too. In this scenario, the elimination of the leukemia cells takes longer than in B-cell leukemias and is associated with a higher risk profile. 


\section{Conflict of interest statement}

Professor Schrappe has received, and continues to receive, third-party funding from the German Cancer Aid. He has received conference delegate fees and fees from continuing medical educational events, as well as travel and hotel expenses from Medac and Eusapharma. He has received lecture honoraria and ringfenced funds into a third-part account from Medac. Professor Bartram has received third-party funding for projects from the German Research Foundation and the German Cancer Aid. Dr Schrauder and Dr Köhler declare that no conflict of interest exists.

Manuscript received on 12 January 2012, revised version accepted on 31 May 2012

Translated from the original German by Dr Birte Twisselmann.

\section{REFERENCES}

1. Riehm H, Gadner $H$, Henze $G$, et al.: Results and significance of six randomized trials in four consecutive ALL BFM trials. Haematol Blood Transf 1990; 33: 439-50.

2. Conter V, Aricò M, Basso G, et al.: Long-term results of the Italian Association of Pediatric Hematology and Oncology (AIEOP) Studies 82, $87,88,91$ and 95 for childhood acute lymphoblastic leukemia. Leukemia 2010; 24: 255-64.

3. Pui CH, Evans WE: Treatment of acute lymphoblastic leukemia. N Engl J Med 2006; 354: 166-78.

4. Moghrabi A, Levy DE, Asselin B, et al.: Results of the Dana-Farber Cancer Institute ALL Consortium Protocol 95-01 for children with acute lymphoblastic leukemia. Blood 2007; 109: 896-904.

5. Aricò M, Valsecchi MG, Rizzari C, et al.: Long-term results of the AlEOPALL-95 Trial for Childhood Acute Lymphoblastic Leukemia: insight on the prognostic value of DNA index in the framework of Berlin-FrankfurtMuenster based chemotherapy. J Clin Oncol 2008; 26: 283-9.

6. Möricke A, Zimmermann M, Reiter A, et al.: Long-term results of five consecutive trials in childhood acute lymphoblastic leukemia performed by the ALL-BFM study group from 1981 to 2000. Leukemia 2010; 24: 265-84.

7. Reiter A, Schrappe M, Ludwig WD, et al.: Chemotherapy in 998 unselected childhood acute lymphoblastic leukemia patients. Results and conclusions of the multicenter trial ALL-BFM 86. Blood 1994; 84: 3122-33.

8. Gaynon PS, Desai AA, Bostrom BC, et al.: Early response to therapy and outcome in childhood acute lymphoblastic leukemia: a review. Cancer 1997; 80: 1717-26

9. Dördelmann M, Reiter A, Borkhardt A, et al.: Prednisone response is the strongest predictor of treatment outcome in infant acute lymphoblastic leukemia. Blood 1999; 94: 1209-17.

10. Brüggemann M, Schrauder A, Raff T, et al.: European Working Group for Adult Acute Lymphoblastic Leukemia (EWALL); International BerlinFrankfurt-Münster Study Group (I-BFM-SG). Standardized MRD quantification in European ALL trials: proceedings of the second international symposium on MRD assessment in Kiel, Germany, 18-20 September 2008. Leukemia 2010; 24: 521-35.

11. Bassing $\mathrm{CH}$, Swat W, Alt FW: The mechanism and regulation of chromosomal V(D)J recombination. Cell 2002; 109 (Suppl): 45-55.

12. Hansen-Hagge TE, Yokota S, Bartram CR: Detection of minimal residua disease in acute lymphoblastic leukemia by in vitro amplification of rearranged T-cell receptor delta chain sequences. Blood 1989; 74: 1762-7.

13. Yamada M, Hudson S, Tournay 0 , et al.: Detection of minimal disease in hematopoietic malignancies of the B-cell lineage by using third-complementarity-determining region (CDR-III)-specific probes. Proc Natl Acad Sci (U SA) 1989; 86: 5123-7.

14. van Dongen JJ, Seriu T, Panzer-Grümayer ER, et al.: Prognostic value of minimal residual disease in acute lymphoblastic leukaemia in childhood. Lancet 1998; 352: 1731-8.
15. Cavé $\mathrm{H}$, van der Werff ten Bosch J, Suciu S, et al.: Clinical significance of minimal residual disease in childhood acute lymphoblastic leukemia N Engl J Med 1998; 339: 591-8.

16. Flohr T, Schrauder A, Cazzaniga G, et al.: International BFM Study Group (I-BFM-SG). Minimal residual disease-directed risk stratification using real-time quantitative PCR analysis of immunoglobulin and T-cell receptor gene rearrangements in the international multicenter trial AIEOP-BFM ALL 2000 for childhood acute lymphoblastic leukemia. Leukemia 2008; 22: 771-82.

17. Pongers-Willemse MJ, Seriu T, Stolz F, et al.: Primers and protocols for standardized detection of minimal residual disease in acute lymphoblastic leukemia using immunoglobulin and T cell receptor gene rearrangements and TAL 1 deletions as PCR targets: report of the BIOMED-1 CONCERTED ACTION: investigation of minimal residual disease in acute leukemia. Leukemia 1999; 13: 110-8.

18. van der Velden VH, Cazzaniga G, Schrauder A, et al.: European Study Group on MRD detection in ALL (ESG-MRD-ALL). Analysis of minimal residual disease by lg/TCR gene rearrangements: guidelines for interpretation of real-time quantitative PCR data. Leukemia 2007; 21: 604-11.

19. Conter V, Bartram CR, Valsecchi MG, et al.: Molecular response to treatment redefines all prognostic factors in children and adolescents with B-cell precursor acute lymphoblastic leukemia: results in 3184 patients of the AIEOP-BFM ALL 2000 study. Blood 2010; 22: 3206-14.

20. Möricke A, Reiter A, Zimmermann M, et al.: German-Austrian-Swiss ALL-BFM Study Group. Risk-adjusted therapy of acute lymphoblastic leukemia can decrease treatment burden and improve survival: treatment results of 2169 unselected pediatric and adolescent patients enrolled in the trial ALL-BFM 95. Blood 2008; 111: 4477-89.

21. Schrappe M, Valsecchi MG, Bartram CR, et al.: Late MRD response determines relapse risk overall and in subsets of childhood T-cell AL: results of the AIEOP-BFM-ALL 2000 study. Blood 2011; 118: 2077-84.

22. Borowitz MJ, Devidas M, Hunger SP, et al.: Clinical significance of minimal residual disease in childhood acute lymphoblastic leukemia and its relationship to other prognostic factors: a Childrens' Oncology Group study. Blood 2008; 111: 5477-85.

23. Stow $P$, Key $L$, Chen $X$, et al.: Clinical significance of low levels of minimal residual disease at the end of remission induction therapy in childhood acute lymphoblastic leukemia. Blood 2010, 115: 4657-63.

24. Bassan R, Spinelli 0, Oldani E, et al.: Improved risk classification for risk-specific therapy based on the molecular study of minimal residual disease (MRD) in adult acute lymphoblastic leukemia (ALL). Blood 2009: 113: 4153-62.

25. Gökbuget N, Kneba M, Raff T, et al : Adults with acute lymphoblastic leukemia and molecular failure display a poor diagnosis and are candidates for stem cell transplantation and targeted therapies. Blood, 2012 (in press).

26. Moreton P, Kennedy B, Lucas G, et al.: Eradication of minimal residual disease in B-cell chronic lymphocytic leukemia after alemtuzumab therapy is associated with prolonged survival. J Clin Oncol 2005; 23 2971-9.

27. Buccisano F, Maurillo L, Del Principe Ml, et al.: Prognostic and therapeutic implications of minimal residual disease in acute myeloid leukemia. Blood 2012; 119: 332-41.

28. Helhlmann, R, Hochhaus A, Baccarani M: Chronic myeloid leukaemia. Lancet 2007; 370: 342-50.

29. Riethdorf S, Wikman H, Pantel K: Biological relevance of disseminated tumor cells in cancer patients. Int J Cancer 2008; 123: 1991-2006.

\section{Corresponding author}

Prof. Dr. med. Claus R. Bartram

Institut für Humangenetik, Universität Heidelberg

Im Neuenheimer Feld 366, 69120 Heidelberg

cr.bartram@med.uni-heidelberg.de 